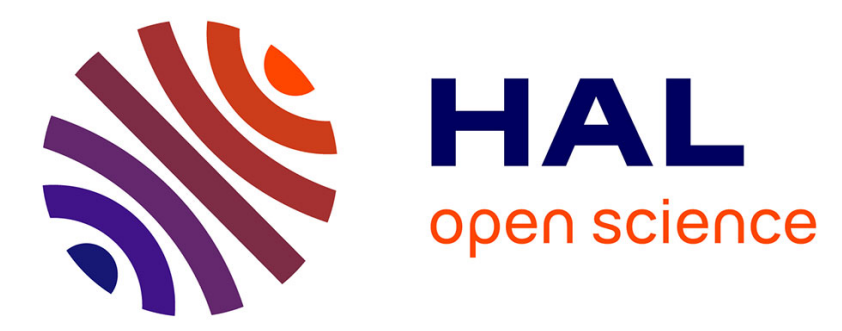

\title{
A DTN routing scheme for quasi-deterministic networks with application to LEO satellites topology
}

Rémi Diana, Emmanuel Lochin, Laurent Franck, Cédric Baudoin, Emmanuel Dubois, Patrick Gelard

\section{- To cite this version:}

Rémi Diana, Emmanuel Lochin, Laurent Franck, Cédric Baudoin, Emmanuel Dubois, et al.. A DTN routing scheme for quasi-deterministic networks with application to LEO satellites topology. IEEE 76th Vehicular Technology Conference: VTC2012-Fall, Sep 2012, Québec, Canada. hal-00868858

\section{HAL Id: hal-00868858 https://hal.science/hal-00868858}

Submitted on 2 Oct 2013

HAL is a multi-disciplinary open access archive for the deposit and dissemination of scientific research documents, whether they are published or not. The documents may come from teaching and research institutions in France or abroad, or from public or private research centers.
L'archive ouverte pluridisciplinaire HAL, est destinée au dépôt et à la diffusion de documents scientifiques de niveau recherche, publiés ou non, émanant des établissements d'enseignement et de recherche français ou étrangers, des laboratoires publics ou privés. 


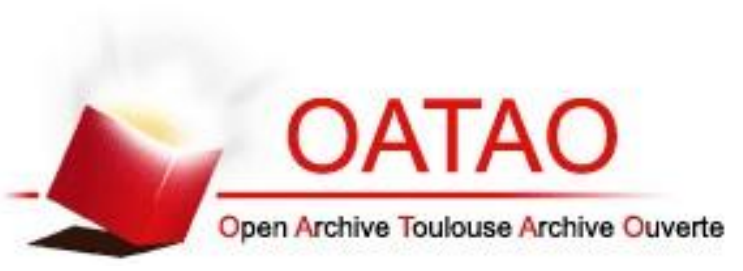

\section{Open Archive Toulouse Archive Ouverte (OATAO)}

OATAO is an open access repository that collects the work of Toulouse researchers and makes it freely available over the web where possible.

This is an author-deposited version published in: http://oatao.univ-toulouse.fr/ Eprints ID: 6236

To cite this document: Diana, Rémi and Lochin, Emmanuel and Franck, Laurent and Baudoin, Cédric and Dubois, Emmanuel and Gelard, Patrick A DTN routing scheme for quasi-deterministic networks with application to LEO satellites topology. (2012) In: IEEE 76th Vehicular Technology Conference: VTC2012Fall, 3-6 Sep 2012, Québec City, Canada.

Any correspondence concerning this service should be sent to the repository administrator: staff-oatao@inp-toulouse.fr 


\title{
A DTN routing scheme for quasi-deterministic networks with application to LEO satellites topology
}

\author{
Rémi Diana ${ }^{1,3,4}$, Emmanuel Lochin ${ }^{1}$, Laurent Franck $^{2}$, Cedric Baudoin ${ }^{3}$, Emmanuel Dubois $^{4}$, Patrick Gelard ${ }^{4}$ \\ ${ }^{1}$ Université de Toulouse, ISAE, TeSA, Toulouse, France \\ ${ }^{2}$ Telecom Bretagne - Institut Telecom, TeSA, Toulouse, France \\ ${ }^{3}$ Thales Alenia Space, Toulouse, France \\ ${ }^{4}$ CNES Toulouse, France
}

\begin{abstract}
We propose a novel DTN routing algorithm, called DQN, specifically designed for quasi-deterministic networks with an application to satellite constellations. We demonstrate that our proposal efficiently forwards the information over a satellite network derived from the Orbcomm topology while keeping a low replication overhead. We compare our algorithm against other well-known DTN routing schemes and show that we obtain the lowest replication ratio without the knowledge of the topology and with a delivery ratio of the same order of magnitude than a reference theoretical optimal routing.

Index Terms-DTN routing, satellite constellations
\end{abstract}

\section{INTRODUCTION}

Satellite constellations have a dynamic character in terms of mobility and connectivity. However, their dynamics are mostly predictable and static routing, enabled by a pre-computation of the network routes, can be applied to them [15][7]. The main drawback of pre-computed routing schemes is to be static and not tolerant to link outages. Other dynamic routing approaches, such as MANET routing, cannot be applied to all satellite constellations as most of the nodes might be intermittently connected. Furthermore, common DTN routing are not efficient as they have been initially designed to perform over random topologies.

DTN was initially created for interplanetary networks [2] but received a great success for opportunistic networks [6]. Several DTN routing schemes have been proposed for social and random networks [16][13][3][10], however the idea of using DTN routing algorithms over LEO satellite constellations is quite new. The quasi-deterministic character of a satellite constellation makes current DTN routing proposals (specifically designed for random networks) not optimal over such specific topologies. We qualify a satellite topology as quasi-deterministic since the traffic nature and link outages might compromise the inherent deterministic character of the constellation. In particular, in Section IV, we show that current implemented DTN routing schemes have a too high replication overhead for satellite networks where the cost of transmission must be taken into consideration. Existing proposals attempt to improve the delivery throughput based on the multicommodity theorem [11]. The principle behind, which extends the definition of the max-cut min-flow theorem [8], is to build a graph by analysing a given quasi-deterministic topology in order to find the maximum achievable throughput that the network can handle [9]. Other schemes, such as Contact Graph Routing [4], propose to compute the optimal route for DTN messages forwarding. However, all these techniques are based on a pre-computed algorithm, require a high computation effort and are not fault tolerant.

We assume a LEO constellation without storage in the payload and no inter-satellite links either. End-to-end communications are done in a multi-hop manner from gateways to gateways via satellites. This illustrates the intermittent character of the chosen topology since gateways are not always covered by a satellite. Over such a LEO constellation, precomputed routing algorithms are often used. These algorithms compute routing tables of each gateway for a given duration $\left[t_{0}, t_{0}+\Delta_{t}\right]$. Thus, routing tables are fixed during $\Delta_{t}$ and will not be recomputed even if an outage occurs during this period. Basically, a pre-computed algorithm corresponds to an implementation of an optimal algorithm if no failures occur and there is no buffer limitation. As a consequence, such a routing scheme is not resistant to link failure. If a link is down during a time before $\Delta_{t}$, the delay to detect it, to compute new routing tables and to upload them in each node might result in several data losses.

In this paper, we propose to cope with this periodic computation scheme problems by designing an optimal DTN replication-like routing scheme for such quasi-deterministic networks. We focus on the optimal number of replication without too much delay compromise and we test our routing protocol over the Orbcomm topology [1]. The rationale of using Orbcomm is linked to its intermittent connectivity. This explains why this topology is best suited for users who send very small amounts of data and where services are much like email and messages based (as over DTN networks) [1]. In Section II, we drive an analysis of the contacts and intercontacts duration of Orbcomm nodes. Following this analysis we propose a novel DTN routing algorithm. We evaluate our proposal against other well-known DTN routing algorithms in Section IV and in a more realistic scenario in Section V. Finally we conclude and discuss this work in Section VI. 


\section{ANALysis OF THE CONSTELlation}

The objective of this section is to characterize the studied topology in terms of contacts and inter-contacts duration between gateways and their distribution. We also assess the inter-gateway availability. The study presented below is driven over a bipartite network: a constellation of satellites and a set of terrestrial gateways. For the sake of simplicity, we first consider that the terrestrial gateways are uniformly distributed (a more realistic scenario will be detailed Section V). Based on Orbcomm, the constellation is formed by 35 satellites distributed over six orbital planes. Three orbital planes contain eight satellites and one contains seven satellites all uniformly spaced. A polar and a retrograde orbital plane each contains two satellites. In this study, the terrestrial gateways are uniformly spaced on Earth with an interval $d=20^{\circ}$. The whole network is presented in Table I and Fig. 1.

TABLE I

CONSTELLATION CHARACTERISTICS

\begin{tabular}{|c|c|c|c|}
\hline Orbital plane & Inclination & Altitude $(\mathrm{km})$ & \# of Satellites \\
\hline 1 & $45^{\circ}$ & $\approx 827$ & 8 \\
\hline 2 & $45^{\circ}$ & $\approx 827$ & 8 \\
\hline 3 & $45^{\circ}$ & $\approx 827$ & 8 \\
\hline 4 & $45^{\circ}$ & $\approx 827$ & 7 \\
\hline Polar & $70^{\circ}$ & $\approx 744$ & 2 \\
\hline Retrograde & $108^{\circ}$ & $\approx 833$ & 2 \\
\hline
\end{tabular}

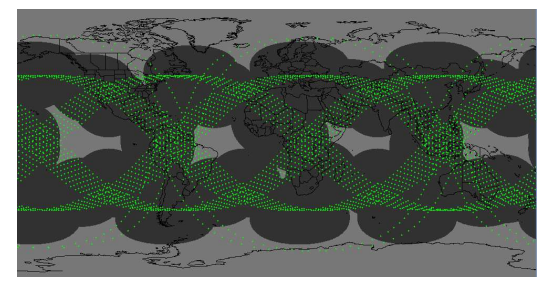

Fig. 1. Orbital traces and footprints

We now present the hypotheses used to analyse this topology.

\section{A. Main hypothesis}

We consider that there is no storing data buffer inside the satellites and no inter-satellite link (ISL). Thus, each satellite is considered as a simple data link between two terrestrial gateways. When a link is available, a contact between two terrestrial gateways is enabled and is defined by a contact duration. All data are carried between the terrestrial gateways where, in our experimental scenarios, some of them are senders while others are receivers. Note that each gateway has the knowledge of the geographical position of all other gateways.

\section{B. Contacts and inter-contacts duration characteristics}

We first illustrate the gateway service availability which corresponds to the distribution of the immediately reachable locations around a gateway. Fig. 2 gives a representation of this repartition. For each figure, the light point corresponds to the reference gateway and the dark surface represents the reachable area. The darker the surface, the more frequent the contact with the gateway. We define the availability as the ratio between the average contact duration and the total duration. An availability of 1 means that the gateway and the remote location are permanently connected. However, this figure does not provide the exact repartition of contact duration and intercontact duration according to the localisation of the gateway. Indeed, this is only an average. Nevertheless, a low availability corresponds generally to a low average contact duration and a long average inter-contact duration.

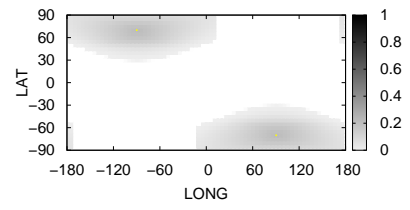

(a)

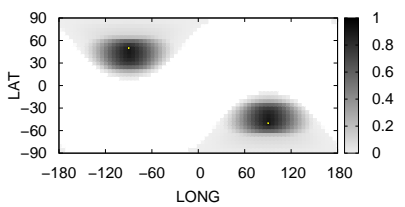

(c)

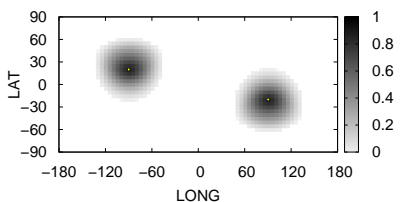

(e)

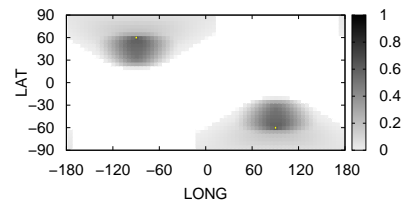

(b)

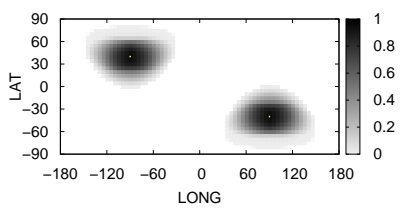

(d)

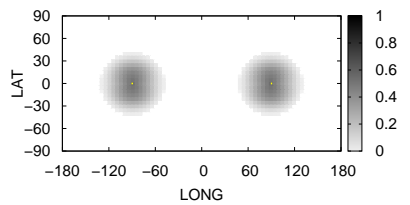

(f)
Fig. 2. Spatial and temporal distribution of the contacts around a gateway (two separate gateways are represented in each figure).

\section{Determination of the routes}

We now focus on the routing aspects and evaluate the possible existing paths. This evaluation is mandatory to better design our routing algorithm. We have chosen two representative gateways, one sender that continuously sends data while the other receives them. Each of the 162 gateways replicates in an epidemic manner the data sent. Then, we look at the routes taken by each data according to their sending time. Fig. 3 gives the results obtained at four different starting times. Thanks to a clustering algorithm, we have identified the five best clusters of routes in terms of delay for a given starting time. For instance, Fig. 3(a) gives five set of routes, with $r_{1}$ representing a set of $n$ routes that have the same and best delay and same path minus one or two hops. Respectively, the next Figures 3(b), 3(c), 3(d) repeat the process for a different starting time spaced by 1000 seconds. 
This experiment allows to conclude that the fastest routes are not static and time dependent. Another aspect underlined by Fig. 3 and particularly by Fig. 3(d) is the fact that there is no spatial and temporal correlation. As this is not the purpose of the paper and due to space constraints, we do not present results that analyse the resilience time (i.e. lifetime) of the fastest route which is anyway very low.

In summary and following several experiments, we have concluded that the lifetime of the routes and the intermittent character of the links prevent the use of standard mobile network routing such as AODV [14] or OLSR [5] that would allow the topology to be fault tolerant. In a general manner, the design of a MANET routing protocol is not adapted over such topology and a DTN approach must be preferred. Following this analysis, in the next section we design a new DTN routing scheme that takes advantage of the topology, comparing it to well-known DTN routing proposals.

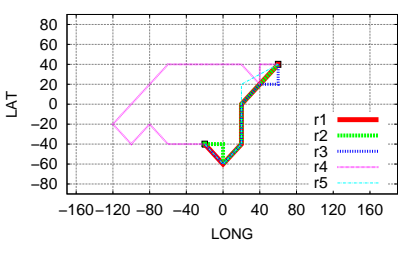

(a)

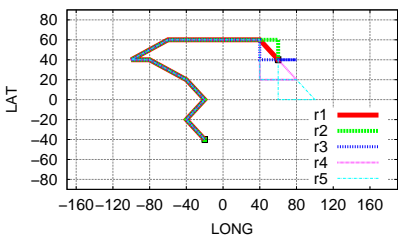

(c)

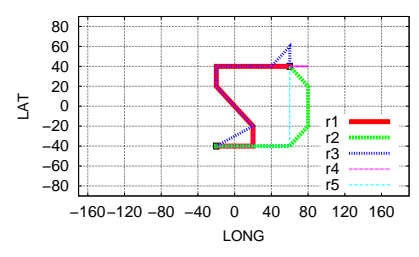

(b)

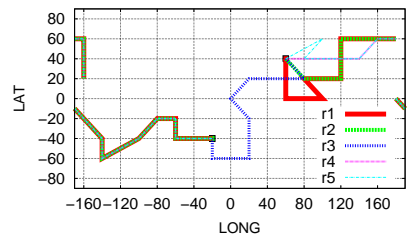

(d)
Fig. 3. Five best routes between two gateways at time $\mathrm{t}=t_{0}, t_{0}+1000$, $t_{0}+2000, t_{0}+3000$

\section{Algorithm PROPOSED}

We rely on the previous analysis in order to design an efficient dynamic routing algorithm without pre-computing. The developed algorithm has to be able to determine every kind of routes, from the shortest to particular routes such as the ones illustrated in Fig. 3(d). Note that we seek to be failure resistant, this means that even if a connection between two gateways falls down, data must be delivered over an alternative path at the expense of an increase of the delivery time. We note that each route goes either eastbound or westbound. In the example of Fig. 3(d), the fastest routes are all westbound (note that this property is linked to the Orbcomm constellation topology and can be changed). Taking this property into account, we develop a replication-like algorithm. We recall that end-toend communications are done in a multi-hop manner from gateways to gateways via a satellite.

The proposed algorithm, DQN, is detailed in Algorithm 1. The beginning of this pseudo code (line \#3) deals with the case where the gateway $R$ is the source. In this case, the

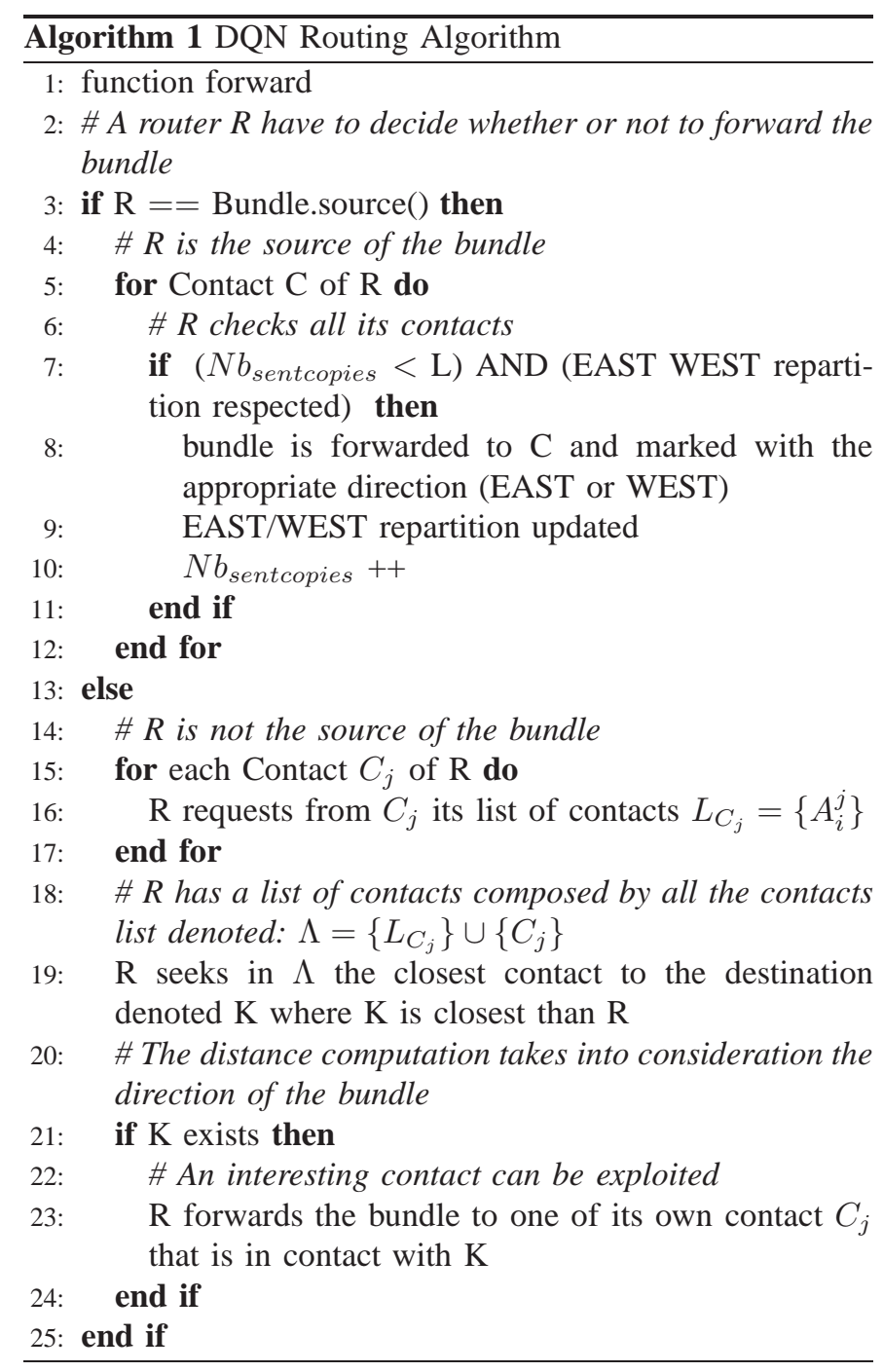

source sprays $L$ copies (also called bundle messages [2]) while trying to respect the balance between west and east in order to increase the chance to discover one of the five fastest routes. Then, no further replication is done. Otherwise, if $R$ is an intermediate gateway which gets one bundle message, it takes a forwarding decision as follows:

1) $R$ requests the current contacts list from all its immediate contact gateways $C_{i}$ (line \#15 in Algorithm 1), to build a list of achievable gateways (i.e. as illustrated in Fig. 4);

2) after retrieving a set of achievable gateways $\left\{A_{i}^{j}\right\}$, the intermediate gateway decides to forward to one of its immediate contact which is also in contact with the $A_{i}^{j}$ gateway the closest to the destination (line \#19);

3 ) if the intermediate gateway does not find any consistent forwarding gateway, it keeps the copy and wait a fixed period in order to obtain a better list of contacts.

\section{Performance evaluation}

In this section, we give a first evaluation of DQN algorithm. In order to provide a consistent analysis, we evaluate the 


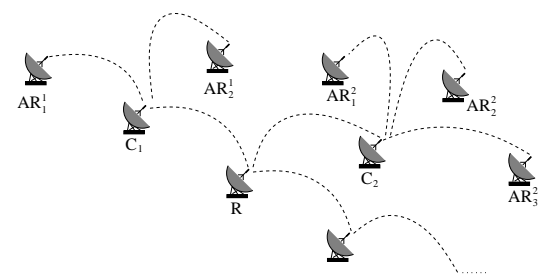

Fig. 4. Illustration of the forwarding decision

performance of our proposal against other similar DTN routing algorithms.

There are two classes of DTN routing algorithms. Those that only forward copies (e.g. hot-potato routing) and those that replicate a set of infinite or limited copies. As our algorithm is a replication-like algorithm, we compare our proposal to Epidemic, Binary Spray and Wait (BSW), PRoPHET and RAPID algorithms.

Epidemic was chosen to give a lower bound of the delay. This scheme has the biggest overhead in terms of number of disseminated copies but is the fastest in terms of time delivery considering that the buffer of the topology are infinite. We use the Epidemic scheme as the reference scheme for delay. Binary Spray and Wait was chosen because it is a popular and efficient DTN routing algorithm which limits the number of replicates. Despite of being optimal for purely random networks, we may expect poor results from this scheme over a quasi-deterministic topology. In our context, due to the per-hop communication scheme, the relative diversity of each node is low and the average route length is high. Moreover, BSW requires at least a $2^{n}$ replication factor to follow a route with $n$ intermediate nodes. As the average route length is high, BSW will need a high replication factor to correctly perform and obtain good delivery ratio performance. For instance, to be able to follow an eight intermediary hops path, the replication factor must be greater than 256, yielding to a high overhead and even not adapted to a satellite constellation.

The advantage of PRoPHET algorithm is to detect particular contact schemes. This makes it a strong candidate to our proposal. However, the performance of this protocol is quite sensitive to its initial parameters $\alpha, \beta, \gamma$ [12].

Finally, we choose RAPID which is based on a per-message and a global utility computation, because this scheme is known to obtain fair performance in terms of delivery delay.

In order to drive a representative simulation, we select four different pairs of nodes. Each pair corresponds to a given source and destination and generates a traffic of 220 bundles spaced by 1000 seconds. We fix $L$ to five in DQN. Results are presented in Fig. 5. For each routing algorithm, we plot the end-to-end delay, defined as the time between the creation of the bundle and the reception of the first replicate by the destination. We also represent the average overhead in terms of number of replicates. The per-bundle overhead, $O_{b}$ is defined for each bundle $b$ as follows:

$$
O_{b}=\frac{n_{t, \text { algo }}-n_{t, o p t}}{n_{t, o p t}}
$$

with $n_{t, \text { algo }}$ the number of bundle transmissions for the selected routing algorithm and $n_{t, o p t}$ the number of bundle transmissions in the optimal case. Note that the Epidemic scheme is the best case in terms of delay but the worst in terms of replicates overhead. The direct competitor to our proposal seems to be RAPID. However, the most important result is that in terms of overhead, our proposal obtains the best score compared to all other schemes.

Previous results provide the average delay. However, it is important to also compare these algorithms with their delay repartition. An algorithm might display similar performance than another on average but can present either a very short or very long delay. To drive this comparison and to complement these results, Fig. 5 gives the CDF of delays. If we consider a compromise between delay and overhead in terms of number of replicated messages, our proposal displays the best tradeoff. It highly decreases the overhead, at least by $35 \%$, which is the most important aspect in a satellite context and obtains a delivery delay only about $20 \%$ higher compared to RAPID. Finally and to better assess the performance of our proposal, a percentage gain balance is plotted in Fig. 6. This figure sums up the whole results provided in Fig. 5. A positive value means that our proposition offers better performances than a given scheme (the gain is relative, $-1 \leqslant G \leqslant 1$ ).

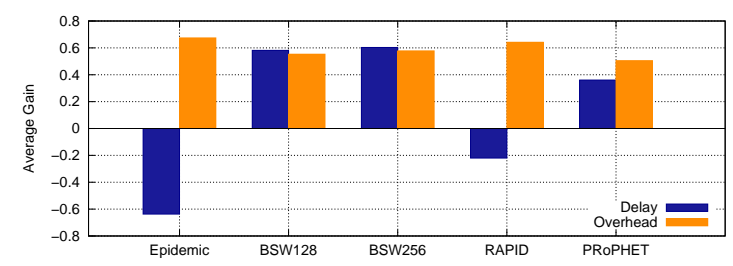

Fig. 6. Gains results against DQN

\section{EXPERIMENT IN A REALISTIC SCENARIO}

We propose in this section to evaluate and compare our algorithm over a more realistic scenario. Thus, we define a topology of 33 gateways placed near major towns. Fig. 7 presents their localization. The main characteristic of this topology is to present isolated gateways. We propose to further optimize the message delivery by analysing the new contacts distribution resulting from this sparse repartition and to enable a contact learning phase in order to discover these isolated gateways. The objective is to prevent spurious replications in areas where the destination would never be reachable.

Fig. 8 presents the results obtained and show that our algorithm outperforms RAPID in this context. The overhead observed is low while the delay is in the worst case, 35\% higher than the optimal delay. This experiment is a simple example that will be further studied in a future work.

\section{CONCLUSION}

We propose in this paper a novel DTN routing algorithm specifically designed for quasi-deterministic networks. The main objective is to propose an alternative to pre-computed 


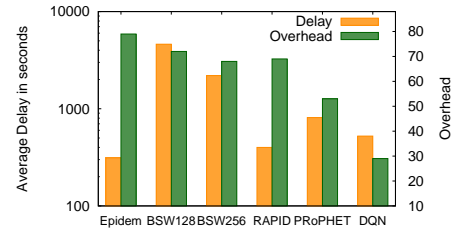

(a) From Ushuaia to Hudson Bay

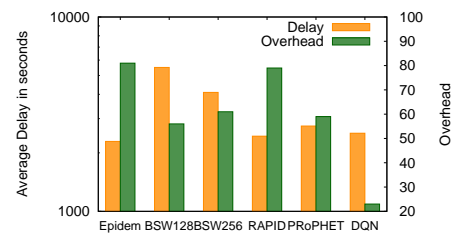

(e) From Leticia to Mokoku

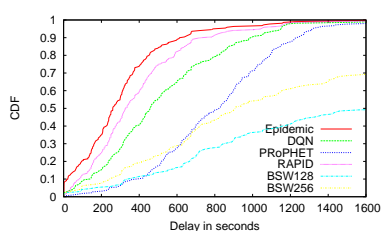

(b) From Ushuaia to Hudson Bay

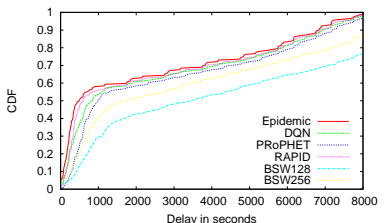

(f) From Leticia to Mokoku

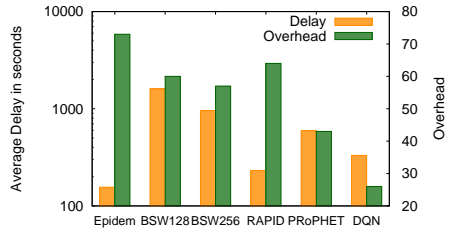

(c) From Santiago to Rome

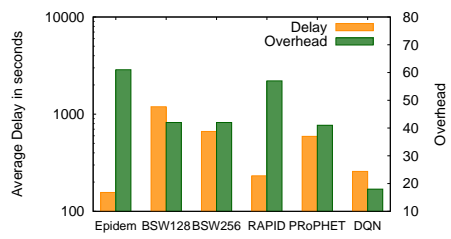

(g) From Istanbul to São Paulo

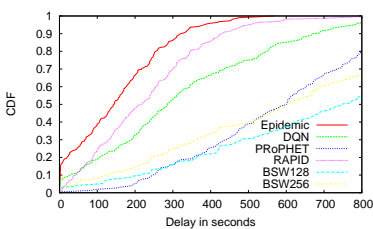

(d) From Santiago to Rome

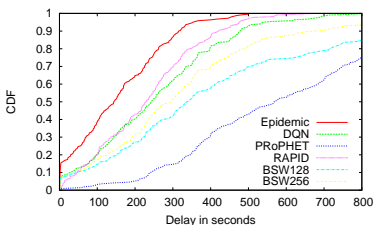

(h) From Istanbul to São Paulo

Fig. 5. Performance evaluation as a function of the delivery delay and the data overhead

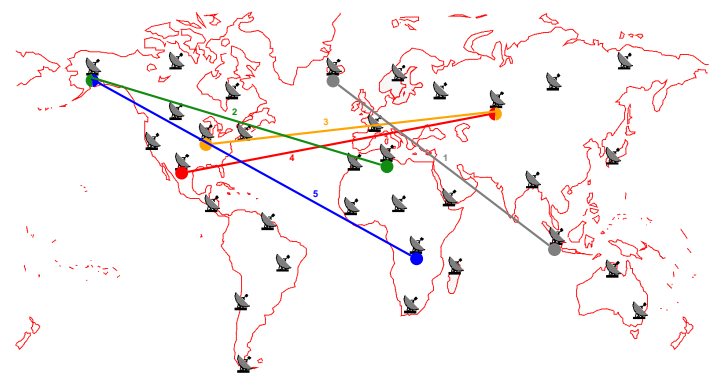

Fig. 7. Topology used and source/destination localization

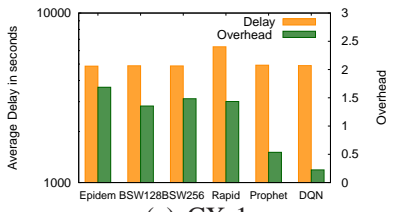

(a) CX 1

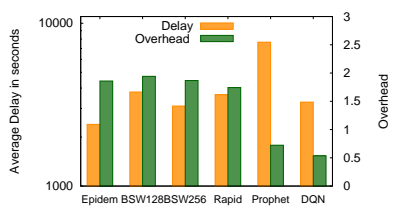

(c) $\mathrm{CX} 3$

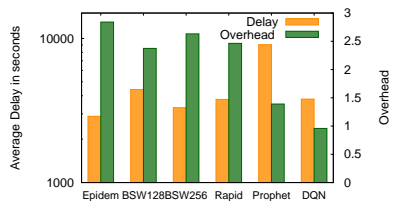

(b) CX 2

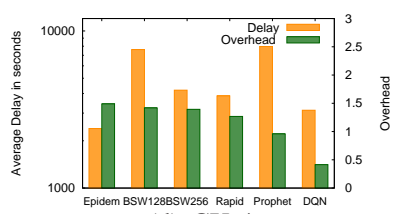

(d) $\mathrm{CX} 4$

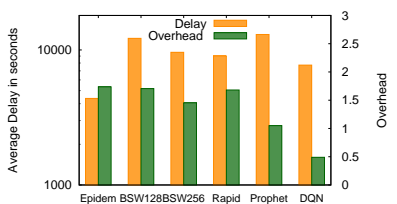

(e) CX 5

Fig. 8. Delay and overhead distribution, each connexion (CX) is represented in Fig. 7

routing scheme commonly used over satellite constellations. We have tested our solution against well-known DTN routing protocols over a topology similar to the Orbcomm constellation. We show that our proposal outperforms in terms of replication overhead most of current DTN routing scheme without too much delay compromise. This makes our solution a potential candidate to enable fault-tolerant routing over satellite constellation.

In a future work, we expect to deeper investigate this scheme in several other scenarios and to investigate further optimizations in the case of isolated gateways.

\section{REFERENCES}

[1] 2009. Orbcomm website, http://www.orbcomm.org/.

[2] V. Cerf and. al. Interplanetary internet (IPN): Architectural definition, 2001. http://www.ipnsig.org/reports/memo-ipnrg-arch-00.pdf.

[3] A. Balasubramanian, B. Levine, and A. Venkataramani. DTN routing as a ressource allocation problem. SIGCOMM, 2007.

[4] S. Burleigh. Contact graph routing. Technical Report draft-burleighdtnrg-cgr-01, IETF, 2010. (Work in progress).

[5] T. Clausen and P. Jacquet. Optimized Link State Routing Protocol (OLSR), 2003.

[6] J. Crowcroft, E. Yoneki, P. Hui, and T. Henderson. Promoting tolerance for delay tolerant network research. SIGCOMM, 2008.

[7] H. Cruz-Sanchez, L. Franck, and A.-L. Beylot. Precomputed routing in a store and forward satellite constellation. In IEEE VTC, 2007.

[8] L. R. Ford and D. R. Fulkerson. Maximal flow through a network. Canad. J. Math., 1956.

[9] P. Giaccone and D. Hay. Optimal routing and scheduling for deterministic delay-tolerant networks. In IEEE WONS, 2009.

[10] P. Hui, J. Crowcroft, and E. Yoneki. Bubble rap: social-based forwarding in delay tolerant networks. In ACM MobiHoc, 2008.

[11] T. Leighton and S. Rao. Multicommodity max-flow min-cut theorems and their use in designing approximation algorithms. Journal of the ACM, 1999.

[12] A. Lindgren, A. Doria, E. Davies, and S. Grasic. Probabilistic routing protocol for intermittently connected networks. Internet Draft draft-irtfdtnrg-prophet-09, Internet Engineering Task Force, 2011.

[13] L. Pelusi, A. Passarella, and M. Conti. Opportunistic networking: data forwarding in disconnected mobile ad hoc networks. Comm. Mag., 2006.

[14] Charles E. Perkins and Elizabeth M. Royer. Ad hoc on-demand distance vector routing. In ACM Hotmobile, 1999.

[15] S.R. Pratt, R.A. Raines, C.E. Fossa, and M.A. Temple. An operational and performance overview of the iridium low earth orbit satellite system. Communications Surveys Tutorials, IEEE, 1999.

[16] T. Spyropoulos, K. Psounis, and C. S. Raghavendra. Spray and wait: An efficient routing scheme for intermittently connected mobile networks. SIGCOMM WDTN, 2005. 